\title{
Research on the Outlook and Solutions in the Development of
}

\author{
Marathon Series in China \\ Long di Dai ${ }^{{ }^{*}} \&$ Si han Zhang ${ }^{2}$ \\ ${ }^{1}$ College of Physical Education, Anhui Normal University, Wuhu, Anhui Province, 241000, China \\ ${ }^{2}$ Department of business administration, Anhui Business Vocational College, Hefei, 230000, China \\ *Long di Dai, E-mail: 932479193@qq.com
}

Received: October 28, 2017 Accepted: November 12, 2017 Online Published: November 16, 2017 doi:10.22158/wjssr.v4n4p350

URL: http://dx.doi.org/10.22158/wjssr.v4n4p350

\begin{abstract}
Today, Marathon Series rise gradually and grow fast, and their development brings undeniable influences, even can drive the development of a city in every aspect, playing an incredible catalytic role in the regional development. The author deeply explored the status and laws of the development of Marathon Series in our country through literature material laws, field investigation and expert interview survey and believes: Marathon Series can even stimulate enthusiasm among common people in running a marathon, can enhance communication and connection between cities, can better promote balanced development between each territory and plays a proactive role in the establishment of Marathon brand; for the future development of Marathon Series in our country, we should vigorously implement "Internet plus" strategies adapt to the era, prepare cooperation programs for the race well, make a plan as sufficiently as possible, actively carry out innovation and realize sustainable development, with a view to contributing fresh strength to the profound and long-lasting development of marathon business and even sport industry in our country as well as the Healthy China 2030 initiative.
\end{abstract}

\section{Keywords}

Marathon, Series, Development Prospect, Development Countermeasures

\section{Analysis of Status}

Obviously, Marathon fascinates today's world. Marathon Series are a new race system and a combination of marathon races with united brands and united themes that are successively held in different substations across various cities in the whole province or all provinces nationwide for a period of time. In our country, one of relatively famous marathon is “China Mountain Marathon Series” firstly launched in 2016 and hosted by the Mountaineering Administration Center of the General Administration of Sport of China and the Chinese Mountaineering Association, with the theme of 
“Runs Towards the Mountains, an Extraordinary Road” and six preliminary rounds planned through the year, to hope mass runners challenge themselves and feel the beauty of mountains and rives and the humanities style of China; the "One Belt, One Road” Marathon Series, whose hosting period is from 2016 to 2018, is China’s first international IP race jointly launched by the Chinese Athletic Association and Wisdom Holdings Group, with domestic first station Baoan, Shenzhen and foreign first station Kuala Lumpur, Malaysia as a place of departure, countries along the "One Belt, One Road” strategies as a substation, such as the major cities in the countries like The United Arab Emirates, Singapore and Maldives. The meaning of the races is to comprehensively upgrade the international image of China's Marathon and make Marathon become the exchange and communication bridge between countries; "Run China" Marathon Series co-sponsored by the Chinese Athletic Association and CCTV Sports Channel in April, 2017 were planned to last one year, with 32 cities for primary rounds like Beijing, Shanghai and Jinlin respectively as a host place, and "Red Tour", "Reform and Opening Up" "Ecosystem Upgrade” and "One Belt, One Road" as their themes. The four themes demonstrate the valuable historical accumulation and spiritual quality of the Chinese nation give a powerful voice of contemporary sport participants and have an impressive domestic and even international influence. Thus, it can be seen that, the influence of Marathon Series has progressively become outstanding, plus, the Several Opinions on Accelerating the Development of the Sports Industry to Promote Sports Consumption issued by the State Council in 2014 clearly defined the National Fitness as a national strategy and put forward the goal of China's sport industry reaching 5 trillion Yuan by 2025. Current social trends can called a absolute spring for Marathon. Therefore, Marathon Series that are progressing and growing surely will have a brilliant future.

\section{Outlook of Marathon Series in Our Country}

\subsection{It Can Even Stimulate Enthusiasm among Common People in Running a Marathon}

Fundamentally, ordinary people love marathon is because the value provision of marathon races caters to the value requirements of national fitness, economic development and urban construction and fits in with the value requirements of the interactive relationship between governments, departments and citizen. Compared with traditional "One way ticket" marathon races, Marathon Series are unmatched for traditional races in extending enthusiasm among runners. As a benefit to running enthusiast, Marathon Series can fully meet running hobbyists' thorough pursuit of the marathon race, a favorite brand, can make runners to go through the whole series races and achieve a grand slam by challenging each primary round, and practically allows running hobbyists to satisfy their cravings in their favorite brand races. That is, the thirst for accomplishing targets and the push of perfectionism will make runner become emotionally dependent on a rang of primary rounds to different extents, thus escalate their enthusiasm to a higher level in running a marathon. 


\subsection{It Can Enhance Exchange and Communication between Cities}

It is well known that Marathon Series are hold in a mode in which "Authorities at higher levels are like thousands of threads and governments at the community level are the single needle that weaves”. They need partial functions among various provinces and cities to be given a fully play to promote overall perfection of races. Final effects of systematical optimization can be achieved and the effect of " $1+1>2$ " is produced, only when good coordination between each part is provided. This requires full communication and exchange among various territories to make the race features and race culture comes down in one continuous line, with their unique style. As the comprehensive dialogue takes place to hold a series of race successfully, the economy, politics and humanity between various places are bound to be fully integrated and benefit each other.

\subsection{It Can Better Promote Balanced Development between Various Territories}

At current stage, there is a status in marathon race in our country, that is, the population in economically developed areas, which run a marathon, accounts of a bigger proportion, whereas the race track in less developed areas is highly optional but the population there that run marathon take up small proportion. This phenomena fully reflects imbalanced development between regions in our country, and the existence of marathon series can drive the inflow of running enthusiasts in less developed area and introduction of capital, opportunities and advanced culture, and can also play a propaganda role in various race field with local characteristics, thus having an effective driving role in promoting the shrinkage of imbalance development between regions.

\subsection{It Plays an Active Role in the Establishment of Marathon Race Brand}

It is commonly known that the large gap between our country's marathon race and foreign famous races is that famous race not only attract more runners but also play a propaganda role in the city and even the whole country as the card carrier in aspects of the building of race brands, thus make the city become famous due to marathon and marathon to grow due to the city. Marathon series are more far-reaching and lasting than one way race in terms of the establishment of race brands. The brand effects they produce are chaining and extensive. Using the strength of various places to build a marathon brand can achieve the result with half the effort. Compared with traditional road-run marathon, marathon series make themselves better publicize the race brand, themes and host units and cities due to its extension in time and broadness in space. Suck broadcast effects are unmatched for traditional single field marathon.

\section{Certain Suggestions on the Development of Marathon Series in Our Country}

\subsection{Vigorously Implement "Internet Plus" Strategies and Adapt to the Era}

As an overall concept, "Internet plus" has a deep meaning that is to accomplish the upgrade of industries through interconnection of traditional industries. Internet intends to sort out supply-demand relationship through the role of its own properties such as openness, equality and interaction and 
through analysis and integration of big data, and enhances the power of economic development and improve efficiency by modifying the production modes of traditional industries, industrial structure and other contents, in hopes of promoting healthy and orderly development of national economy. Content construction of the network should be enhanced and lasting "adhesive” relationship with runner fans be established in marathon series in our country. The vitality and influence of marathon can be really and appropriately utilized only when both are taken into account. "Internet plus" in nature is deep integration performed between Internet and various traditional industries using information communication technologies and Internet platforms, so as to create a new development ecosystem. It is a new social formation, can give a full play the internet in the optimization and integration of social resources configuration, deeply fuse the innovative results of Internet into various fields of economy and society, improve the productivity and creativity of the whole society and form more extensive new formation of economic development with internet as an infrastructure and an implementation tool. In the "Internet plus" era, it is necessary to use internet thinking to lead the development of sport industries and realize product orientation (drive quality improvement and efficiency enhancement, transformation and upgrade of traditional sport business formation, strengthen the existing value of sport industry), operational upgrade (drive interconnection of sports, enterprise alliance, optimize commercial ecosystem of sport industry), mode innovation (build industrial platform, practice cross-boundary advantages and enhance integration ecosystem of sport industry), and only in this way, the practical consolidation of aspects like integration opportunity and ecosystem reconstruction of internet and sport industries can be realized to make our country's sport industry constantly develop in more advanced and solid direction.

\subsection{Make out Cooperation Program of Races and Plan As Sufficiently As Possible}

Contradiction is unity of opposites and the fundamental power for the development of things. Cooperation program for races, also called race planning proposal, is the embodiment formation of planning results, refers to the text that plan some race in the future and show it to readers, that is, use to existing knowledge to develop imagination and the quickest possible realized goals in a reality where resources are available, with the purpose of presenting planning thinking and contents objectively, clearly, and vividly, and efficiently guiding practical actions. General cooperation program for races is divided into proposing stage (file a proposal with target customers, and file a proposal with internal organization), feasible stage (overview of proposal, environmental analysis, establishment of organizational relationship and selection and analysis of operational mode), and executive stage, and three tricks "precision”, "simplification” and "depth” should be followed. Host parties for marathon series are more required to make out marathon cooperation program compared with single field races, and the contents typically include outline of race (race program, race position, race highlights), warm-up propaganda (news release conference, launch ceremony, online interaction), efficiency analysis (media efficiency, propaganda efficiency, social efficiency, economic efficiency), introduction 
of resources for holding a race, registration channel, media resources, case introduction and other parts.

\subsection{Proactive Innovation, Sustainable Development}

Innovation is a soul for the progress of a nation and an inexhaustible driving force for the prosperity and development of a nation. To realized innovation, the only way is to make runners run for running and generate value and meaning by running, and only in this way, the fundamental goal of innovation can be met. Actually, since the beginning of 2011, "Benefit draws many responses" was launched in Beijing Marathon to make running action for charity. Now Beijing Marathon becomes a leader in China's marathon and even the charity projects in China and races. It builds a platform for marathon, and allows charities to get involved as a protagonist in the drama, integrates charity into running, uses sport to converge human power and makes contribution to the development of charity business. In 2015, 1,705 players from 17 institutions totally participated in the activity of "run for charity" and raised a charity fund of 2,791,965 Yuan. All the charity funds were used to support deployment and activity contents of projects of charity organizations. The main supporting and fund raising mode are donation, financial assistance and crowd funding by large enterprises, kind net friends and runners, etc. Therefore, constant innovation in every aspect of marathon races surely will be the trend of holding races across various places in our country. With the increasing popularization of marathon series in our country economic, political and cultural innovations with marathon series as a platform will make marathon race in our country become consistently vigorous and flourishing and allow themselves to develop on a lasting and profound basis, with innovation as a springboard, and remain popular.

\section{Conclusion}

Marathon series are in initial development stage and move on in exploration. We can make them struggle to forge ahead in constant standardization only when we face the opportunity of development of the era with adequate patient and confidence, and we can constantly discover problems and resolve problems and find a correct way for the optimization of marathon series in our country, can make them more personalized, reasonable, diversified, innovative, and can better enrich research results of sports industry, promote discipline development, improve discipline knowledge system, lay solid foundation for the construction of sports power and health China, and make marathon series contribute to the development of economy, politics and culture in our country only when key breakthrough points are found and system optimization is performed.

\section{References}

Beijing marathon official website. (2015). Starting from the heart of modern Beijing-Sidelights on the 2015 Beijing marathon "run for public welfare" [EB/OL]. Retrieved from http://www.beijing-marathon.com/html/page-6172.html

Huang. C. X., \& Wang, D. (2015). "Internet plus” means what deep understanding of the "Internet 
plus”. News and writing, 2015(05), 5-9.

Liu, Y. F. (2017). "Internet plus” under the background of ecological analysis and construction of the sports industry. Journal of Nanjing Sport college (SOCIAL SCIENCES EDITION), 31(03), 34-39.

The State Council. (2014). Some opinions of the State Council on speeding up the development of sports industry and promoting sports consumption.

Zhang, X. L. (2016). Value Analysis of "Hot" Phenomenon in Chinese Marathon Race. Journal of Shenyang Sport University, 2016(04), 118-122. 